\section{Prevalencia de rinitis alérgica en poblaciones de varios estados de México}

\begin{abstract}
RESUMEN
Antecedentes: la rinitis alérgica es una enfermedad inflamatoria de la mucosa nasal, caracterizada por síntomas de prurito, rinorrea, congestión nasal y estornudos, inducidos por una respuesta mediada por IgE. En México tenemos reportes de prevalencia de rinitis alérgica con variaciones de 5 a $48 \%$ cuando se pregunta si hubo síntomas de rinitis los últimos 12 meses.
\end{abstract}

Objetivo: determinar la prevalencia de rinitis alérgica en población escolar de varios estados de México.

Material y método: estudio descriptivo de prevalencia en el que se aplicó un cuestionario a población preescolar, primaria, secundaria y preparatoria. Se realizó en cuatro ciudades de cuatro estados: Puebla, Puebla, Tulancingo, Hidalgo, Tlaxcala, Tlaxcala y Cancún, Quintana Roo. Los padres contestaron los cuestionarios de preescolar y primaria y los alumnos de secundaria y preparatoria contestaron sus cuestionarios. El estudio se realizó de junio de 2014 a enero de 2015. El instrumento utilizado fue el Cuestionario diagnóstico de rinitis alérgica para estudios epidemiológicos.

Resultados: de las encuestas aplicadas se obtuvieron 8,159 cuestionarios contestados; en la ciudad de Puebla: 2,267; Tulancingo, Hidalgo: 2,478; Tlaxcala, Tlaxcala: 2,574; Cancún, Quintana Roo: 840; 4,190 (51\%) correspondieron al sexo masculino. El porcentaje promedio general de prevalencia de rinitis alérgica entre los cuatro estados, incluidas todas las edades, fue de $15 \%$.

Conclusiones: con la aplicación del cuestionario diagnóstico de rinitis alérgica para estudios epidemiológicos en las cuatro ciudades de cuatro estados diferentes encontramos una prevalencia de rinitis alérgica de 15\% en mayores de 13 años y de 13\% en niños de 12 años o menos.

Palabras clave: rinitis alérgica, prevalencia, cuestionario.

\section{Prevalence of rhinitis allergic in populations of several states of Mexico}

\section{ABSTRACT}

Background: Allergic rhinitis is an inflammatory disorder of the nasal mucosa, characterized by symptoms of itching, rhinorrhea, nasal congestion and sneezing induced by an IgE-mediated response. In Mexico we have reports of prevalence, with fluctuations of $5.5 \%$ to $47.7 \%$ with the question of rhinitis symptoms the past 12 months.
Eleazar Mancilla-Hernández ${ }^{1}$ Miguel Alejandro Medina-Ávalos² Raúl Humberto Barnica-Alvarado ${ }^{1}$ Diego Soto-Candia ${ }^{3}$ Rosario Guerrero-Venegas ${ }^{1}$ Yahvéh Zecua-Nájera ${ }^{1}$

${ }^{1}$ Colegio Mexicano de Inmunología Clínica y Alergia A.C.

2 Servicio de Alergia, Hospital Regional Veracruz, Instituto de Seguridad y Servicios Sociales de los Trabajadores del Estado, Veracruz, Veracruz.

${ }^{3}$ Servicio de Inmunología Clínica y Alergia, Hospital General, Instituto de Seguridad y Servicios Sociales de los Trabajadores del Estado, Tlaxcala, Tlaxcala.

Recibido: 22 de enero 2015

Aceptado: 30 de abril 2015

Correspondencia: Dr. Eleazar Mancilla Hernández Acrópolis 5101

72567 Puebla, Puebla, México

manele05@yahoo.com.mx

Este artículo debe citarse como

Mancilla-Hernández E, Medina-Ávalos MA, BarnicaAlvarado RH, Soto-Candia D y col. Prevalencia de rinitis alérgica en poblaciones de varios estados de México. Revista Alergia México 2015;62:196-201. 
Objective: To determine the prevalence of allergic rhinitis in schoolchildren from various states of Mexico.

Material and method: A descriptive study of prevalence in which a questionnaire was applied to preschool, elementary-, middle- and high-school population. It was performed in four cities in four states of Mexico: Puebla, Puebla, Tulancingo, Hidalgo, Tlaxcala, Tlaxcala and Cancun, Quintana Roo. Parents answered questionnaires of preschool and elementary school and middle- and high-school students answered their questionnaires. The study was conducted from June 2014 to January 2015. The instrument used was: questionnaire diagnosis of allergic rhinitis for epidemiological studies.

Results: Of the surveys, 8,159 completed questionnaires were obtained, in the city of Puebla: 2,267, Tulancingo, Hidalgo: 2,478, Tlaxcala, Tlaxcala: 2,574, Cancun, Quintana Roo: 840; total male: 4,190 (51\%). The overall average rate of prevalence of allergic rhinitis among four states including all respondents ages was 15\%.

Conclusions: With the use of the questionnaire diagnosis of allergic rhinitis for epidemiological studies in the four cities in four different states, we found a prevalence of allergic rhinitis of $15 \%$ in $\geq 13$ yearpopulation and $13 \%$ in $\leq 12$ year-old children.

Key words: allergic rhinitis, prevalence, questionnaire.

\section{ANTECEDENTES}

La rinitis alérgica es una enfermedad inflamatoria de la mucosa nasal, caracterizada por síntomas de prurito, rinorrea, congestión nasal y estornudos, inducidos por una respuesta mediada por IgE. ${ }^{1}$ La mucosa nasal tiene un alto grado de vascularidad arterial subepitelial y sinusoides cavernosos venosos, lo que es una característica clave en los síntomas y los cambios en la vasculatura pueden causar obstrucción nasal severa. ${ }^{2}$ En la inmunopatogénesis inflamatoria participan mediadores químicos, las células Th2, citocinas, quimiocinas y moléculas de adhesión en la mucosa respiratoria. ${ }^{3}$ En pocos minutos después del contacto con los alergenos las células cebadas sensibilizadas con IgE se degranulan liberando mediadores preformados y sintetizados de novo; estos mediadores causan los síntomas característicos de rinorrea, estornudos, congestión nasal y prurito, la fase temprana se atribuye a la liberación de leucotrienos, prostaglandinas, histamina y citocinas. La histamina se ha demostrado virtualmente como la productora de todos los síntomas de fase temprana en la rinitis alérgica a través de su unión a los receptores H1.4,5

La rinitis alérgica es la enfermedad atópica más frecuente en Estados Unidos; más de 80 millones de personas padecen rinitis alérgica, con síntomas nasales y oculares durante más de siete días al año. Los costos socioeconómicos son similares en la rinitis alérgica, porque afectan el rendimiento escolar, la socialización y la productividad en el trabajo. ${ }^{6}$ En México tenemos estudios de prevalencia de la rinitis alérgica en diferentes estados utilizando el cuestionario de ISAAC; en Ciudad Guzmán, Jalisco, encuestaron a niños de 6 a 12 años de edad, reportando 5\% de rinitis alérgica. ${ }^{7}$ En la ciudad de Durango en 
población general se encontró una prevalencia de $8 \% .{ }^{8}$ Un estudio epidemiológico realizado en cuatro ciudades: Ciudad Victoria, Mexicali, Monterrey y Tijuana, en población escolar de seis a siete años y de 13 a 14 años de edad, encontró una prevalencia de $24 \%$ de síntomas de rinitis los últimos 12 meses. ${ }^{9}$ En la Ciudad de México en población escolar de 6-7 y de 13-14 años se encontró una prevalencia de $4-5 \%$ con la pregunta de diagnóstico de rinitis alérgica, con la pregunta de los síntomas acumulativos de rinitis se obtuvo 40 a $55 \%$ y con la pregunta de síntomas de rinitis actuales, 29 a $48 \% .{ }^{10}$ Otro estudio realizado en la Ciudad de México sin utilizar metodología ISAAC, se investigó la prevalencia de enfermedades alérgicas usando definiciones operacionales basadas en los síntomas de las enfermedades alérgicas; se encontró $20 \%$ de rinitis alérgica. ${ }^{11}$ En Cuernavaca, Morelos, en población escolar de 6-8 años y de 11-14 años, la prevalencia del diagnóstico médico de rinitis fue de $5 \%$, de los síntomas de rinitis en los últimos 12 meses de $10 \%$ en niños de de 6-8 años y de $10 \%$ en adolescentes de 11 a 14 años. ${ }^{12}$ En este trabajo utilizamos el cuestionario diagnóstico de rinitis alérgica para estudios epidemiológicos. ${ }^{13}$

El objetivo de la investigación fue determinar la prevalencia de rinitis alérgica en poblaciones de varios estados de México.

\section{MATERIAL Y MÉTODO}

Estudio descriptivo de prevalencia, realizado en escuelas particulares y oficiales, con previa autorización de la Secretaría de Educación Pública (SEP) de cada estado, la asignación de escuelas la hizo la SEP y las encuestas se aplicaron a población preescolar, primaria, secundaria y preparatoria. Se realizó en cuatro ciudades de cuatro estados: Puebla, Puebla, Tulancingo, Hidalgo, Tlaxcala, Tlaxcala y Cancún, Quintana Roo. Los padres contestaron los cuestionarios de los alumnos de preescolar y primaria y los alum- nos de secundaria y preparatoria contestaron sus cuestionarios. El estudio se realizó de junio de 2014 a enero de 2015. El instrumento utilizado fue el cuestionario diagnóstico de rinitis alérgica para estudios epidemiológicos.

Para el análisis estadístico se obtuvieron promedios, porcentajes y desviación estándar.

\section{RESULTADOS}

De las encuestas aplicadas se obtuvieron 8,159 cuestionarios contestados, con la siguiente distribución: en la ciudad de Puebla: 2,267; Tulancingo, Hidalgo: 2,478; Tlaxcala: 2,574 y Cancún: 840; el total del sexo masculino fue de 4,190 (51\%). El porcentaje promedio general de prevalencia de rinitis alérgica entre los cuatro estados incluidas todas las edades encuestadas fue de $15 \%$. La distribución de prevalencia por ciudad y por sexo de los alumnos de secundaria y preparatoria se observan en el Cuadro 1.

El promedio general de prevalencia de rinitis alérgica en los escolares de 13 años o más en las cuatro ciudades fue de $15 \%$. La frecuencia por sexo entre la población con rinitis alérgica en las cuatro ciudades fue: en Puebla: $30 \%$ sexo masculino, $70 \%$ sexo femenino; Tulancingo: $41 \%$ sexo masculino, 59\% sexo femenino; Tlaxcala: $39 \%$ sexo masculino, $61 \%$ sexo femenino; Cancún: $43 \%$ sexo masculino, $67 \%$ sexo femenino. El promedio general en las cuatro ciudades con diagnóstico de rinitis alérgica fue de $38 \%$ para el sexo masculino y de $62 \%$ para el sexo femenino.

La distribución de prevalencia por ciudad y la frecuencia por sexo en alumnos de preescolar y primaria se observan en el Cuadro 2.

El promedio general de prevalencia de rinitis alérgica en población de 12 años o menos, preescolares y escolares de primaria, fue de $13 \%$ en las cuatro ciudades. La frecuencia por sexo en cada 
Mancilla-Hernández E y col. Prevalencia de rinitis alérgica

Cuadro 1. Prevalencia de rinitis alérgica. Distribución por ciudad y sexo de los alumnos de secundaria y preparatoria

\begin{tabular}{lcccc}
\hline Ciudad & Encuestados & Rinitis alérgica & Sexo masculino & Sexo femenino \\
Puebla, Puebla & 1,687 & $254(15 \%)$ & $77(5 \%)$ & $177(10 \%)$ \\
Tulancingo, Hidalgo & 1,419 & $232(16 \%)$ & $96(7 \%)$ & $.136(9 \%)$ \\
Tlaxcala, Tlaxcala & 1,303 & $88(7 \%)$ & $35(3 \%)$ & $53(4 \%)$ \\
Cancún, Quintana Roo & 364 & $79(22 \%)$ & $34(9 \%)$ & $45(12 \%)$
\end{tabular}

Cuadro 2. Prevalencia de rinitis alérgica. Distribución por ciudad y sexo de los alumnos de preescolar y primaria

\begin{tabular}{lcccc}
\hline Ciudad & Encuestados & Rinitis alérgica & Sexo masculino & Sexo femenino \\
\hline Puebla, Puebla & 580 & $88(15 \%)$ & $56(10 \%)$ & $32(5 \%)$ \\
Tulancingo, Hidalgo & 1,059 & $186(17 \%)$ & $101(9 \%)$ & $85(8 \%)$ \\
Tlaxcala, Tlaxcala & 1,271 & $107(8 \%)$ & $44(3 \%)$ & $63(5 \%)$ \\
Cancún, Quintana Roo & 476 & $69(14 \%)$ & $35(7 \%)$ & $34(7 \%)$
\end{tabular}

ciudad, considerando exclusivamente los casos de rinitis alérgica, tuvo la siguiente distribución: en Puebla: $63 \%$ sexo masculino, $37 \%$ sexo femenino; Tulancingo: $54 \%$ sexo masculino, $46 \%$ sexo femenino; Tlaxcala: $41 \%$ sexo masculino, 59\% sexo femenino; Cancún: 51\% sexo masculino, $49 \%$ sexo femenino. El promedio general por sexo en las cuatro ciudades fue de $52 \%$ para el sexo masculino y de $48 \%$ del sexo femenino.

El promedio de edad de los encuestados de 13 años o más fue de 14.4 \pm 1.9 años, los resultados de la frecuencia en porcentajes con la que ocurrió la rinitis alérgica de acuerdo con la edad de estos alumnos, correspondientes a secundaria y preparatoria, se comunican en la Figura 1.

El promedio de edad en los encuestados de 12 años o menos fue de 8.6 \pm 2.5 años. La distribución de la frecuencia por edad de estos niños, que corresponden a preescolares y escolares de primaria, se comunican en la Figura 2.

\section{DISCUSIÓN}

El porcentaje promedio de prevalencia de alergia en alumnos de 13 años o más y en los de 12 años

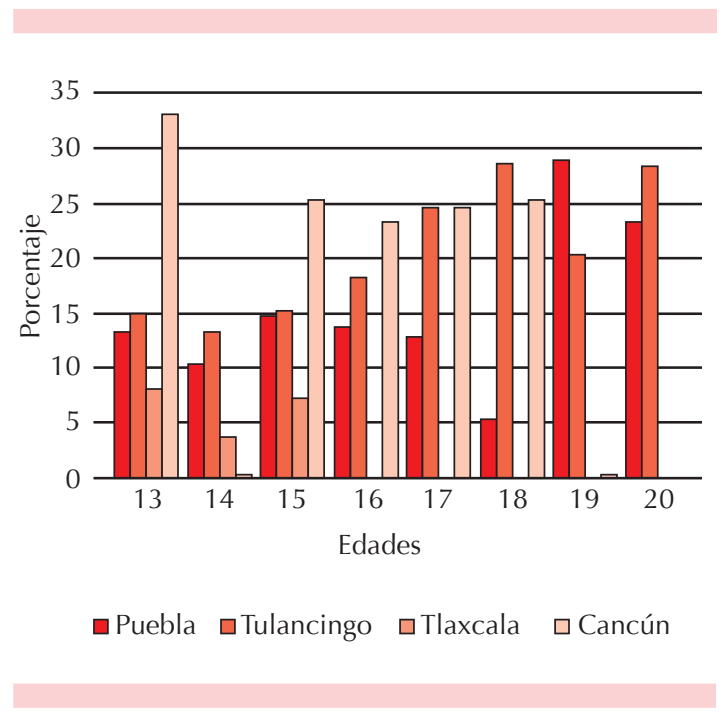

Figura 1. Prevalencia de rinitis alérgica en escolares de 13 años o más.

o menos es cercano en su resultado entre ambos: de 15 y $13 \%$, respectivamente; los resultados de prevalencia encontrados con este cuestionario fueron más altos que los reportados en Ciudad Guzmán, Durango y Cuernavaca, que varían entre 5 y $10 \%{ }^{7,8,12}$ y más bajos que las prevalencias reportadas en cuatro ciudades del norte de México y de la Ciudad de México, que varían 


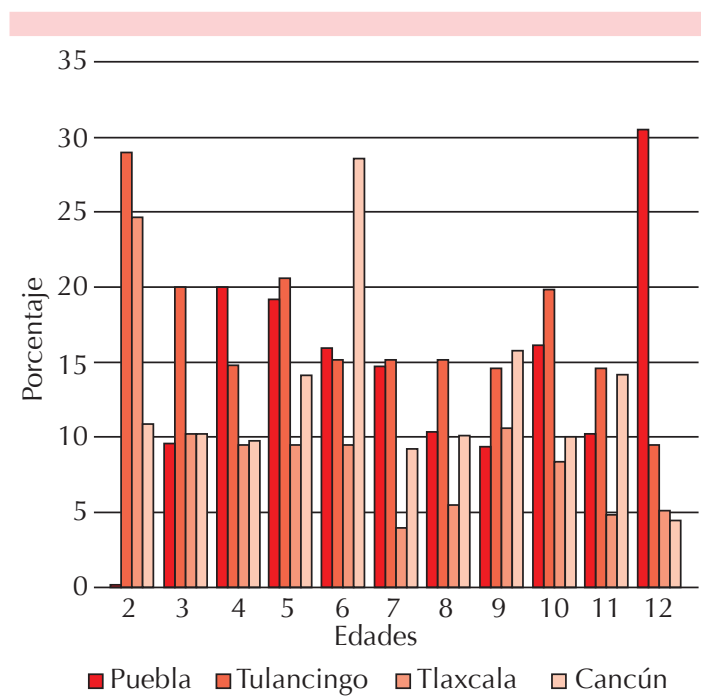

Figura 2. Prevalencia de rinitis alérgica en escolares de 12 años o menos.

entre 24 y $47 \%$, respectivamente. ${ }^{9,10}$ Todos los estudios con los que comparamos nuestros resultados utilizando el cuestionario diagnóstico de rinitis alérgica para estudios epidemiológicos ${ }^{13}$ se realizaron con metodología ISAAC.

El sexo femenino predominó en los pacientes con rinitis alérgica, en los de 13 años o más en términos generales y en los de 12 años o menos predominó ligeramente el sexo masculino. En la población escolar de 13 años o más, el mayor porcentaje promedio de rinitis alérgica se obtuvo en la ciudad de Tulancingo, Hidalgo, con $21 \%$, seguida de Cancún 19\%, Puebla 15\% y Tlaxcala 6\%. La tendencia promedio de rinitis alérgica en los niños de 12 años o menos fue similar: Tulancingo $17 \%$, Puebla con $14 \%$, Cancún $13 \%$ y Tlaxcala 10\%. Los promedios obtenidos por ciudades por edades $\geq 13$ y $\leq 12$ años variaron un poco con respecto a los obtenidos en los cuadros, porque estos últimos reportan los promedios de los cuestionarios contestados por los padres de alumnos de preescolar y primaria en donde hay alumnos hasta de 12 años, y de los cuestionarios contestados directamente por los alumnos de secundaria y preparatoria, en primer año de secundaria también hay alumnos de 12 años.

\section{CONCLUSIONES}

Con la utilización del cuestionario diagnóstico de rinitis alérgica para estudios epidemiológicos en las cuatro ciudades de cuatro estados diferentes encontramos una prevalencia de rinitis alérgica de $15 \%$ en adolescentes de 13 años o más y de $13 \%$ en niños de 12 años o menos. El promedio general de rinitis alérgica fue de $38 \%$ para el sexo masculino y de $62 \%$ para el sexo femenino en los mayores de 13 años, y de $52 \%$ para el sexo masculino y de $48 \%$ del sexo femenino en los menores de 12 años.

\section{REFERENCIAS}

1. Howarth PH. ABC of allergies: pathogenic mechanisms: a rational basis for treatment. BMJ 1998;316:758-761.

2. Bousquet J, Vignola A, Leynaert B, Demoly P. Links between rhinitis and asthma. Allergy 2003;58:733-41.

3. Bousquet J, Vignola AM, Campbell AM, Michel FB. Pathophysiology of allergic rhinitis. Int Arch Allergy Immunol 1996;110:207-218.

4. White MV, Kaliner MA. Mediators of allergic rhinitis. J Allergy Clin Immunol 1992;90:699-704.

5. Togias A. Unique mechanistic features of allergic rhinitis. J Allergy Clin Immunol 2000;105:S599-S604.

6. Adams PF, Hendershot GE, Marano MA. Current estimates from the national health interview survey. Atlanta: Centers for Disease Control and Prevention/National Center for Health Statistics, 1996.

7. Bedolla-Barajas M, Cuevas-Ríos G, García-Barboza E, Barrera-Zepeda AT, Morales-Romero J. Prevalence and associated factors to allergic rhinitis in school children of ciudad Guzman, Mexico. Rev Invest Clin 2010;62:244-251.

8. Cisneros-Pérez V, Alvarado-Esquivel C. Prevalence of allergic rhinitis in Durango, México. Rev Alerg Mex 2004;51:4953.

9. González-Diaz SN, Del Río-Navarro BE, Pietropaolo-Cienfuegos $D R$, et al. Factors associated with allergic rhinitis and adolescents from northern México: International Study of 
Mancilla-Hernández E y col. Prevalencia de rinitis alérgica

Asthma a Allergies in Childhood Phase IIIB. Allergy Asthma Proc 2010;4:53-62.

10. Del-Río-Navarro BE, Luna-Pech JA, Berber A, Zepeda-Ortega A. Factors associated with allergic rhinitis in children from Northern Mexico City. J Investig Allergol Clin Immunol 2007;17:77-84.

11. López-Pérez G, Morfin-Maciel BM, López-López JG y cols. Factores de riesgo relacionados con enfermedades alérgicas en la ciudad de México. Rev Alergia Méx 2010;57:18-25.
12. Tatto-Cano MI, Sanín-Aguirre LH, González V, Ruiz-Velasco $\mathrm{S}$, Romieu I. Prevalence of asthma, rhinitis and eczema in school children in the city of Cuernavaca, Mexico. Salud Pública Mex 1997;39:497-506.

13. Mancilla-Hernández E, Medina-Ávalos MA, Osorio-EscamiIla RE. Validación de un cuestionario diagnóstico de rinitis alérgica para estudios epidemiológicos. Rev Alergia Mex 2014;61:153-161. 